\title{
WHEN THE DISEASE ARISES FROM THE TREATMENT: CORTICOSTEROID-INDUCED PSYCHOSIS A CASE REPORT AND BRIEF LITERATURE REVIEW
}

\author{
Amorim, Maria João *,Maurício, Albuquerque Janaína *, Brandão, Daniela *, Araújo, Filipa ** \\ *Psychiatry Trainee, Psychiatry Department, Unidade Local de Saúde do Alto Minho, Viana do Castelo, Portugal \\ **Psychiatrist, Psychiatry Department, Unidade Local de Saúde do Alto Minho, Viana do Castelo
}

Objectives

This study proposes to clarify the development of corticosteroid-induced psychosis focusing on risk factores, onset timming of symptoms and therapeutic strategy.
Background and aims

Methology

Corticosteroids are a potent therapeutic option in clinical practice in general but they are associated with a large variety of side-effects, especially when used in high doses, such as neuropsychiatric.

The etiopathological mechanism responsible for the onset symptoms remains uncertain. Some studies relate the reduction of seretonine release and the toxicity of these drugs on hippocampal neurons with this symptomatology.
Systematic review on neuropsychiatric effects of corticosteroid treatment using as database: Medline and PubMed, illustrated by a case report with information from clinical interview.
Corticosteroids are used to control and treat a wide range of clinical entities, but are often associated with the onset of adverse effects, including neuropsychiatric symptoms, which occur in about 1,8 to $57 \%$ of patients.

\section{Neuropsychiatric side-effects}

(often severe, unpredictable, even at low doses)

$\checkmark$ Mood disorders (hypomania / mania in early treatment versus depressive symptoms with long-term treatment);

$\checkmark$ Psychosis (almost exclusively at prednisone doses $\geq 20 \mathrm{mg} /$ day);

$\checkmark$ Irritability and Insomnia

Anxious symptomatology

Behavioral changes;

$\checkmark$ Cognitive impairment

Vuicidal ideation / suicidal behaviors (severe cases)

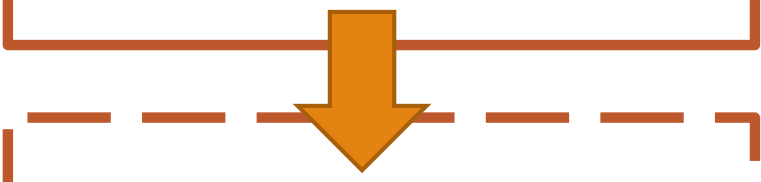

High dose corticosteroids treatments (higher than $40 \mathrm{mg} /$ day of prednisone or equivalent) is associated with a $20 \%$ risk of developing severe psychiatric symptomatology (enough to justify the use of psychotropics similar to those used in the same idiopathic psychopathologies.

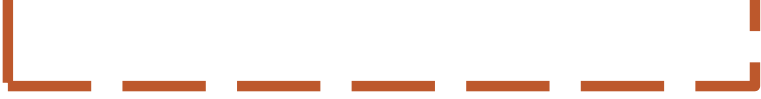

\section{How to proceed}

Stop whenever possible, or reduce $\mathrm{CT}$ dose immediately

(safely and prudently)

Weaning regimens are sufficient to resolve psychotic symptoms in about $94 \%$ of cases

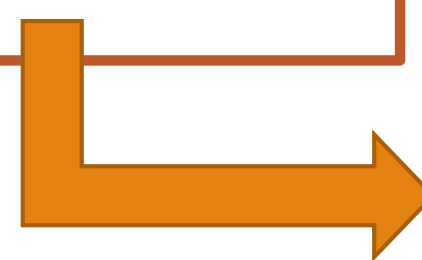

\section{CASE REPORT}

51 year-old, recently diagnosed with breast cancer,

who underwent mastectomy and axillary lymph node

clearence and was under adjuvante chemoterapy (submitted

to a high dose of corticosteroids (> $60 \mathrm{mg} /$ day ) as integral part of the scheme.

The patient checked into the emergency room with behavioral alterations, delusions of mystical and paranoid content, and auditory hallucinations.

Given the absence of psychiatric antecedents, and after exclusion of organic cause, delusional alterations were interpreted as a direct result of corticotherapy, a known cause of the symptomatology described above.

Clinical improvement was observed with the establishment of antipsychotic therapy (haloperidol $5 \mathrm{mg} 1+1+1$ ).

When severe psychotic symptoms psychiatric drugs should be used for symptomatic control.

There are no guidelines and no drugs approved for this purpose. The most described in existing studies: Atypical antipsychotics

Olanzapine $2.5-20 \mathrm{mg} /$ day effective in $92 \%$ of reported cases (resolution of symptoms within 5 weeks)

Risperidone 1 to $4 \mathrm{mg} /$ day And also:

Lithium (0.8-1.2mEq /L) good results in both treatment and prevention
Most important risk factor for developing corticosteroid psychois is the dose of corticosteroids used:

$1.3 \%$ with doses $<40 \mathrm{mg} /$ day $4.6 \%$ with doses between $40-80$ $18.4 \%$ with doses $>80 \mathrm{mg} /$ day

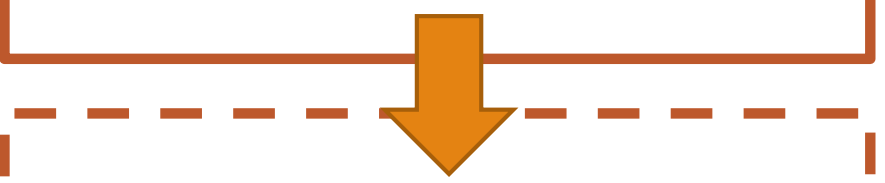

The dose has no predictive value for the onset, severity, duration or type of symptoms. I - - - - - - - - - I

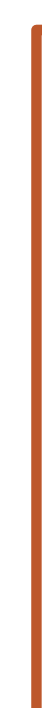

\section{Onset timing \\ May occur at any stage of} treatment (beginning, during and even after termination)

60 to $86 \%$ during the first week of treatment

Up to $90 \%$ in the first 6 weeks of treatment

Excellent prognosis (complete resolution is expected)

Prevention regimens using lithium with good results especially when underlying pathology implies longterm corticosteroid therapy.

\section{Conclusions:}

Due to the significant risk of corticosteroid-induced psychosis and the absence of applicable guidelines, it is important to address this issue, alerting health professionals to the possible therapeutic strategies with antipsychotics and mood stabilizers, outcome and expected symptoms resolution. Most of the existing data are case studies, and due to the lack of guidelines associated with the high prevalence of this type of symptoms associated with corticotherapy, it is essential to carry out studies with statistically significant populations for the establishment and normalization of therapeutic strategies. 